\title{
Long-Term Risk of Progressive Chronic Kidney Disease in Patients with Severe Acute Kidney Injury Requiring Dialysis after Coronary Artery Bypass Surgery
}

\author{
Cynthia C. Lim ${ }^{a} \quad$ Chieh Suai Tan ${ }^{a}$ Cynthia M.L. Chia ${ }^{b}$ Ann Kheng Tan ${ }^{b}$ \\ Jason C.J. Choo ${ }^{a}$ Manish Kaushik $^{a}$ Han Khim Tan ${ }^{a}$ \\ ${ }^{a}$ Department of Renal Medicine, Singapore General Hospital, and ${ }^{b}$ Department of \\ Cardiothoracic Surgery, National Heart Center, Singapore, Singapore
}

\section{Key Words}

Acute kidney injury · Renal replacement therapy · Intensive care · Coronary artery bypass .

Kidney failure $\cdot$ Chronic kidney disease

\begin{abstract}
Aim: Few studies have evaluated patients after cardiac surgery for subsequent chronic kidney disease (CKD) which increases cardiovascular morbidity and mortality. This study aimed to ascertain the long-term renal outcome in adult patients with severe acute kidney injury (AKI) after coronary artery bypass graft (CABG) surgery. Methods: This is a single-center retrospective cohort study of consecutive adult patients who received acute dialysis for AKI after CABG between February 8, 2009 and January 30, 2011. Data on pre- and intra-operative factors were retrieved from electronic medical records. The primary endpoint was CKD progression as defined by dialysis dependence or doubling of serum creatinine from the pre-operative level. Secondary endpoints included in-hospital mortality and renal function at 3 months and 1 year. Results: Fifty-five patients required acute dialysis after CABG. The median age was 67 years (IQR: 61,75 ), and 70.9\% were male. Median pre-operative serum creatinine was 157 $\mu \mathrm{mol} / \mathrm{I}$ (IQR: 122, 203). A total of 19 patients (34.5\%) died. The median follow-up time for hospital survivors was 44.2 months (IQR: 25.0, 49.4) after surgery. Among the 36 survivors, 14 patients (38.9\%) reached the primary endpoint. Patients with CKD progression had higher pre-operative serum creatinine [median $214 \mu \mathrm{mol} / \mathrm{I}$ (IQR: 159, 399) vs. $155 \mu \mathrm{mol} / \mathrm{l}(112,187)$, $\mathrm{p}=0.015$ ] and lower eGFR [median $20.4 \mathrm{ml} / \mathrm{min} / 1.73 \mathrm{~m}^{2}$ (IQR: 11.9, 38.2) vs. $39.9 \mathrm{ml} / \mathrm{min} / 1.73$ $\mathrm{m}^{2}(25.9,55.5), \mathrm{p}=0.027$ ] compared to those who did not have CKD progression. Conclusion: Patients with severe AKI after CABG are at high risk of long-term renal dysfunction and should be monitored regularly for deterioration.




\section{CardioRenal Medicine}

\begin{tabular}{l|l}
\hline Cardiorenal Med 2015;5:157-163 \\
\hline DOI: 10.1159/000381068 & $\begin{array}{l}\text { (c) 2015 S. Karger AG, Basel } \\
\text { www.karger.com/crm }\end{array}$ \\
\hline
\end{tabular}

Lim et al.: Long-Term Risk of Progressive Chronic Kidney Disease in Patients with Severe Acute Kidney Injury Requiring Dialysis after Coronary Artery Bypass Surgery

\section{Introduction}

Cardiovascular disease is the leading cause of mortality worldwide [1]. Among patients with severe or multi-vessel coronary artery disease, coronary artery bypass graft (CABG) improves survival compared to medical revascularisation [2]. However, acute kidney injury (AKI) is common after cardiac surgery, ranging from 10 to $60 \%$ depending on the definition used [3-8]. AKI was associated with greater short-term mortality such that up to $40-60 \%$ of patients with severe AKI died during the hospitalisation $[3,9,10]$. A recent meta-analysis confirmed that patients who required dialysis for AKI post-operatively had greater early mortality [7]. Although some studies have examined long-term mortality and cardiovascular outcomes in survivors of severe AKI after cardiac surgery [6,11-13], few have evaluated the long-term renal outcomes and prognostic factors for chronic kidney disease (CKD) [4]. A systematic review of studies published between 2004 and 2014 by Pickering et al. [7] using consensus definitions for AKI did not find any studies that reported associations between bypass-associated AKI and CKD. As CKD and end-stage renal disease (ESRD) are associated with increased cardiovascular morbidity and mortality, decreased functional capacity and quality of life, as well as increased healthcare and socioeconomic costs [14, 15], we need to identify at-risk individuals for closer monitoring. This study thus aimed to ascertain the incidence and risk factors for adverse long-term renal outcomes in adult patients with severe AKI requiring acute renal replacement therapy (ARRT) after CABG surgery.

\section{Methods}

In this retrospective, Institutional Review Board-approved study (reference number 2008/270/C), patients who underwent on-pump cardiopulmonary bypass (CPB) for CABG and developed severe AKI requiring ARRT between February 2009 and January 2011 were identified using records from the Department of Cardiothoracic Surgery and the Renal Dialysis Center. Patients who were dialysis-dependent prior to surgery or died within $48 \mathrm{~h}$ post-operatively were excluded. Individual medical records were reviewed and information collected included patients' demographics; risk factors for CKD and coronary artery disease such as hypertension, diabetes mellitus and cerebral or peripheral vascular disease; cardiac function including pre-operative myocardial infarction, left ventricular systolic ejection fraction; European System for Cardiac Operative Risk Evaluation (EuroSCORE) [16]; pre-operative need for inotropic or ventilatory support including intra-aortic balloon pump or extracorporeal membrane oxygenation, and pre- and post-operative hemoglobin. Peri-operative data retrieved included need for emergency surgery; concurrent valve surgery; duration of $\mathrm{CPB}$, and need for surgical re-exploration.

Pre-operative serum creatinine was defined as the last recorded serum creatinine value within 1 week before surgery, while post-operative serum creatinine was defined as the peak serum creatinine within $48 \mathrm{~h}$ after surgery. Serum creatinine was measured by the Jaffe method (Unicel DxC 800, Beckman Coulter) calibrated to the National Institute of Standards and Technology Liquid Chromatography Isotope Dilution Mass Spectrometry (LC-IDMS) and expressed in micromoles per liter $(\mu \mathrm{mol} / \mathrm{l})$. Estimated glomerular filtration rate (eGFR) was calculated by the CKD EPI formula which was validated in our local population [17, 18]. AKI was present if post-operative serum creatinine increased $\geq 0.3 \mathrm{mg} / \mathrm{dl}(26.4 \mu \mathrm{mol} / \mathrm{l})$ within $48 \mathrm{~h}$ after the preoperative value, according to the Kidney Disease: Improving Global Outcomes (KDIGO) recommendations [19]. The severity of AKI was graded in accordance to the modified Acute Kidney Injury Network (AKIN) classification using changes in serum creatinine only as hourly urine output data were not available in all patients. Stage 1 was defined as a serum creatinine increment of $>26.4 \mu \mathrm{mol} / \mathrm{l}$ to $1.5-2$ times the pre-operative value; stage 2 if serum creatinine increased to 2-3 times the pre-operative value, and stage 3 if it increased to $>3$ times the pre-operative value [20]. The time from surgery to dialysis initiation and modality of ARRT at initiation [continuous renal replacement therapy (CRRT) or sustained low-efficiency dialysis] were also recorded.

Primary outcome was CKD progression as defined by dialysis dependence for ESRD or doubling of serum creatinine from the pre-operative level. Other outcomes included in-hospital mortality and renal function measured by CKD EPI eGFR at 3 months and at 1 year amongst survivors. Data was censored at the 
Table 1. Baseline demographic, comorbidity and peri-operative characteristics in patients with AKI requiring ARRT after CABG surgery $(\mathrm{n}=55)$

Age, years

Male gender

Diabetes mellitus

Hypertension

Cerebral or peripheral vascular disease

Pre-operative myocardial infarction

Pre-operative left ventricular ejection fraction $\leq 35 \%$

EuroSCORE

Pre-operative inotropic or ventilatory support

Pre-operative hemoglobin, $\mathrm{g} / \mathrm{dl}$

Decrease in post-operative hemoglobin $\geq 20 \%$ from pre-operative level

Emergency surgery

Concurrent valve surgery

CPB duration, min

Surgical re-exploration

Pre-operative serum creatinine, $\mu \mathrm{mol} / \mathrm{l}$

Pre-operative CKD EPI eGFR, $\mathrm{ml} / \mathrm{min} / 1.73 \mathrm{~m}^{2}$

$$
\begin{gathered}
67(61,75) \\
39(70.9) \\
37(67.3) \\
52(94.5) \\
11(20.0) \\
26(47.3) \\
26(47.3) \\
4.7(2.5,12.9) \\
13(23.6) \\
11.4(10.2,13.0) \\
27(49.1) \\
19(34.5) \\
9(16.4) \\
110(83,136) \\
13(23.6) \\
157(122,203) \\
37.5(22.2,49.3)
\end{gathered}
$$

Categorical variables are shown as numbers (percentages) and continuous variables shown as medians (IQR).

last clinic visit, ESRD, or death. IBM SPSS Statistics 21.0 (IBM Corp., Armonk, N.Y., USA) was used for statistical analysis. Categorical variables were presented as percentages, and continuous variables were summarised as medians with interquartile ranges (IQR). Differences between patients with primary outcomes versus those without were analysed using the Fisher's exact test or Mann-Whitney U tests where appropriate. Statistical significance was defined as $\mathrm{p}<0.05$.

\section{Results}

Among 1,489 patients who underwent CABG between February 2009 and January 2011, 55 patients had severe AKI requiring ARRT. Their demographic, comorbidity, and peri-operative clinical parameters are described in table 1 . These patients were generally very unwell prior to surgery, with 13 patients $(23.6 \%)$ requiring inotropic or ventilatory support and one third (34.5\%) requiring emergency bypass. Median pre-operative serum creatinine and eGFR were $157 \mu \mathrm{mol} / \mathrm{l}$ (IQR: 122, 203) and $37.5 \mathrm{ml} / \mathrm{min} / 1.73 \mathrm{~m}^{2}$ (IQR: 22.2, 49.3), respectively. AKI occurred within $48 \mathrm{~h}$ after CABG in 43 patients (78.2\%). Serum creatinine at RRT initiation was available in 53 patients. Twenty-six patients (47.3\%) had AKIN stage 3, $11(20.0 \%)$ had stage 2 , and $16(32.7 \%)$ had stage 1 at the time of RRT initiation. Among 49 patients who had their urine output documented, 48 had oliguria at the time of RRT initiation. The type of RRT modality at initiation was documented in 46 patients. Sustained low-efficiency hemodialysis was the modality at initiation in 17 patients compared to CRRT in 29 patients, of whom 25 received continuous veno-venous hemodialfiltration (CVVHDF).

Figure 1 describes the outcomes of these patients. In-hospital mortality occurred in 19 (34.5\%) patients with AKI requiring RRT after CABG. Thirty-three patients had renal recovery at the time of discharge with median serum creatinine $176 \mu \mathrm{mol} / \mathrm{l}$ (IQR: 126, 252) and CKD EPI eGFR $31.1 \mathrm{ml} / \mathrm{min} / 1.73 \mathrm{~m}^{2}(22.4,47.2)$. The median follow-up time of the hospital survivors was 44.2 months $(25.0,49.4)$ after surgery. Median CKD EPI eGFR 3 and 12 months after surgery were $34.8 \mathrm{ml} / \mathrm{min} / 1.73 \mathrm{~m}^{2}$ (IQR: 20.4, 57.6) and $34.7 \mathrm{ml} / \mathrm{min} / 1.73 \mathrm{~m}^{2}$ (19.6, 
Lim et al.: Long-Term Risk of Progressive Chronic Kidney Disease in Patients with

Severe Acute Kidney Injury Requiring Dialysis after Coronary Artery Bypass Surgery

Fig. 1. Outcomes of patients with severe AKI requiring ARRT after CABG surgery.

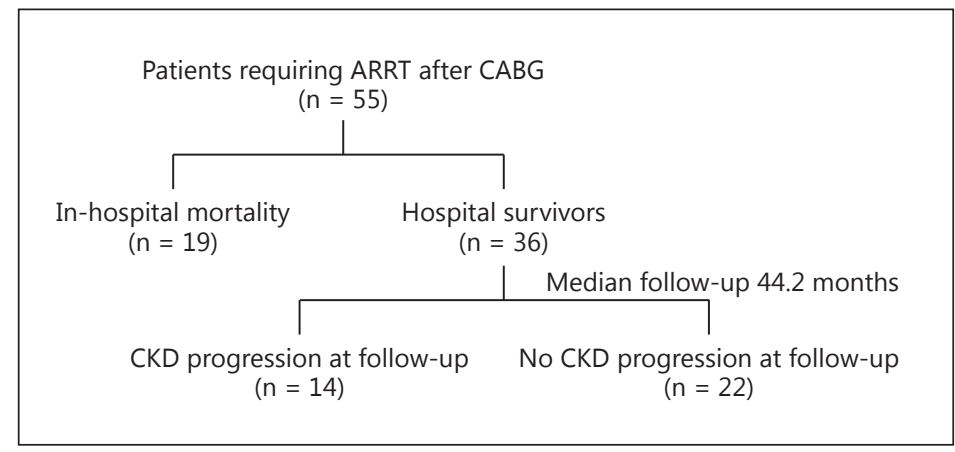

Table 2. Comparison of demographic, comorbidity and peri-operative characteristics in hospital survivors ( $\mathrm{n}=36$ ) with progressive CKD and those without

\begin{tabular}{|c|c|c|c|}
\hline & $\begin{array}{l}\text { Progressive } \\
\text { CKD }(n=14)\end{array}$ & $\begin{array}{l}\text { No progressive } \\
\text { CKD }(n=22)\end{array}$ & $\mathrm{p}$ value \\
\hline Age, years & $68(54,71)$ & $66(61,76)$ & 0.66 \\
\hline Male gender & $9(64.3)$ & $14(63.6)$ & 0.97 \\
\hline Diabetes mellitus & $10(71.4)$ & $18(81.8)$ & 0.68 \\
\hline Hypertension & $14(100)$ & $21(95.5)$ & 1.00 \\
\hline Cerebral or peripheral vascular disease & $4(28.6)$ & $2(9.1)$ & 0.18 \\
\hline Pre-operative myocardial infarction & $6(42.9)$ & $10(45.5)$ & 0.88 \\
\hline Pre-operative left ventricular ejection fraction $\leq 35 \%$ & $8(57.1)$ & $8(36.4)$ & 0.22 \\
\hline EuroSCORE & $3.5(2.2,15.6)$ & $2.9(1.8,5.9)$ & 0.23 \\
\hline Pre-operative inotropic or ventilatory support & $3(21.4)$ & $3(13.6)$ & 0.66 \\
\hline Pre-operative hemoglobin, g/dl & $10.7(10.0,12.4)$ & $12.1(10.2,13.1)$ & 0.49 \\
\hline Decrease in post-operative hemoglobin $\geq 20 \%$ from pre-operative level & $7(50.0)$ & $8(36.4)$ & 0.42 \\
\hline Emergency surgery & $4(28.6)$ & $8(36.4)$ & 0.72 \\
\hline Concurrent valve surgery & $3(21.4)$ & $1(4.5)$ & 0.28 \\
\hline CPB duration, $\min$ & $87(74,122)$ & $105(79,122)$ & 0.28 \\
\hline Surgical re-exploration & $3(21.4)$ & $3(13.6)$ & 0.66 \\
\hline Pre-operative serum creatinine, $\mu \mathrm{mol} / \mathrm{l}$ & $214(159,399)$ & $155(112,187)$ & 0.015 \\
\hline Pre-operative CKD EPI eGFR, ml/min $/ 1.73 \mathrm{~m}^{2}$ & $20.4(11.9,38.2)$ & $39.9(25.9,55.5)$ & 0.027 \\
\hline AKIN stage 3 by creatinine criteria at RRT initiation & $10(71.4)$ & $9(40.9)$ & 0.09 \\
\hline Interval from CABG to ARRT, days & $1(1,3)$ & $1(1,2)$ & 0.87 \\
\hline CRRT at initiation ${ }^{\mathrm{a}}$ & $7(50.0)$ & $10(55.6)$ & 0.76 \\
\hline
\end{tabular}

Categorical variables are shown as numbers (percentages) and compared using Pearson's $\chi^{2}$ test. Continuous variables are shown as medians (IQR) and compared via the Mann-Whitney U test.

${ }^{a}$ ARRT modality at dialysis initiation was available in 32 survivors.

54.1), respectively. Among the 36 survivors, 14 (38.9\%) had CKD progression, of whom 6 had dialysis-dependent ESRD. Table 2 compares the patients who had progressive CKD with those who did not. There were no significant differences between the groups regarding co-morbidities, pre-operative cardiac function, EuroSCORE, or need for inotropic and ventilatory support. Higher pre-operative serum creatinine [median $214 \mu \mathrm{mol} / \mathrm{l}(\mathrm{IQR}: 159,399)$ vs. $155 \mu \mathrm{mol} / \mathrm{l}$ $(112,187), \mathrm{p}=0.015$ ] and lower eGFR [median $20.4 \mathrm{ml} / \mathrm{min} / 1.73 \mathrm{~m}^{2}$ (IQR: 11.9, 38.2) vs. 39.9 $\left.\mathrm{ml} / \mathrm{min} / 1.73 \mathrm{~m}^{2}(25.9,55.5), \mathrm{p}=0.027\right]$ were associated with CKD progression. Although dialysis initiation at AKIN stage 3 was more common among patients with progressive CKD than among those without (71.4 vs. $40.9 \%)$, this was not statistically significant $(p=0.09)$. 


\section{CardioRenal Medicine}

\begin{tabular}{l|l}
\hline Cardiorenal Med 2015;5:157-163 \\
\hline DOI: 10.1159/000381068 & $\begin{array}{l}\text { @ 2015 S. Karger AG, Basel } \\
\text { www.karger.com/crm }\end{array}$ \\
\hline
\end{tabular}

Lim et al.: Long-Term Risk of Progressive Chronic Kidney Disease in Patients with Severe Acute Kidney Injury Requiring Dialysis after Coronary Artery Bypass Surgery

\section{Discussion}

Among this cohort of critically ill patients requiring ARRT after CABG, nearly 80\% developed AKI early within $48 \mathrm{~h}$ after surgery. Cardiac surgery potentiates the risk of ischemic injury and acute tubular necrosis by several mechanisms: the mean arterial pressure in CPB tends to be low, and patients often have hypotensive episodes during surgery from general anesthesia or blood loss. It is also postulated that non-pulsatile flow in CPB disrupts the renal vascular autoregulation and generation of free hemoglobin during CPB causes endothelial injury and subsequent glomerulosclerosis, which leads to CKD [21]. Despite this risk for CKD, there is little data on long-term renal outcomes in the post-cardiac surgery literature [7].

This study showed that among survivors of severe AKI requiring ARRT after CABG, almost half of the patients developed progressive CKD. In a similar single-center retrospective study, Srivastava et al. [22] evaluated 82 hospital survivors who required ARRT after cardiac surgery. At a mean follow-up of 5.2 years, 3 patients (3.6\%) required dialysis and $18(21.9 \%)$ required surveillance by renal physicians because of a deterioration in renal function. Luckraz et al. [23] found that 2 patients (2.2\%) required long-term dialysis among 92 patients who required CRRT after cardiac surgery. However, renal function among dialysis-free survivors was not described in either study $[22,23]$. Factors which appear to predict poorer long-term renal outcome include higher pre-operative serum creatinine and greater post-operative increment in serum creatinine [22, 24]. Ishani et al. [24] retrospectively studied 29,388 patients who underwent cardiac surgery and found that incident CKD (defined as eGFR $<60 \mathrm{ml} / \mathrm{min}$ / $1.73 \mathrm{~m}^{2}$ ) occurred in $25.1 \%$ of patients without post-operative AKI compared to $53.5 \%$ of patients with a $\geq 100 \%$ increase in post-operative serum creatinine level. A recent multicenter Swedish study of 29,330 post-CABG patients confirmed that the ESRD risk was greater in patients with more severe AKI. The adjusted hazard ratio (95\% confidence interval) for ESRD was $2.92(1.87,4.55)$ for AKIN stage 1 and $3.81(2.14,6.79)$ for AKIN stages 2 and 3 [25].

There is increasing interest in determining the optimal timing of ARRT to optimise outcomes for critically ill patients with AKI. Among patients after cardiac surgery, a 'late' ARRT start of $>3$ days post-operatively was associated with increased in-hospital mortality (80.4\%), compared to those who started ARRT within 3 days of surgery $(80.4$ vs. $53.2 \%, \mathrm{p}<$ 0.001) [26]. Using AKIN stage 3 as a definition for 'late' start versus AKIN stages 1 and 2 as 'early start', we did not find an association with mortality or CKD progression. This is consistent with recent studies in critically ill septic AKI patients that found no difference in-hospital mortality when creatinine-based criteria were used to determine ARRT initiation [27, 28]. A change in peri-operative serum creatinine levels in critically ill patients may be less reliable as a biomarker of renal injury as it can be influenced by muscle mass, concurrent sepsis, or hemodilution during surgery [29]. Also, patients who initiate ARRT at milder serum creatinine increments may have concurrent oliguria or fluid overload, which contribute to the need for ARRT. Unfortunately, volume status was not readily available in our retrospective study. Similarly, other retrospective studies on AKI have identified urine output and fluid status as frequently missing data [3].

The dilemma of defining pre-morbid renal function is often encountered in AKI studies. In the absence of consensus criteria, we used the pre-operative serum creatinine value for defining 'baseline' kidney function. This is consistent with other large epidemiological studies on AKI in post-cardiac surgery patients and the large ASSESS-AKI study which defined 'baseline' kidney function based on pre-operative serum creatinine results within 7 days before non-urgent cardiac surgery $[3,30]$. However, we recognise that patients who require emergency surgery after myocardial infarction may have elevated pre-operative serum creatinine compared to their true baseline values. Other limitations of this study include a small study population with possible inadequate power to detect a significant difference. Due to its 
retrospective nature, potential confounders such as indications for initiating ARRT and subsequent nephrology management were not standardized. As this was a single center study and all CABG surgeries were conducted with CPB support, these results may not be generalizable to patients with different risk profiles or centers with different surgical practice such as use of the off-pump technique.

In conclusion, patients with pre-existing renal impairment are at risk of long-term deterioration after surviving severe AKI requiring ARRT after CABG surgery. These individuals will require regular outpatient monitoring with timely nephrology referrals in the event of deterioration.

\section{Disclosure Statement}

The authors confirm that they have no relevant financial interests.

\section{References}

1 Nowbar AN, Howard JP, Finegold JA, et al: 2014 Global geographic analysis of mortality from ischaemic heart disease by country, age and income: statistics from World Health Organisation and United Nations. Int J Cardiol 2014;174:293-298.

2 Deb S, Wijeysundera HC, Ko DT, et al: Coronary artery bypass graft surgery vs percutaneous interventions in coronary revascularization: a systematic review. JAMA 2013;310:2086-2095.

3 Englberger L, Suri RM, Li Z, et al: Clinical accuracy of RIFLE and Acute Kidney Injury Network (AKIN) criteria for acute kidney injury in patients undergoing cardiac surgery. Crit Care 2011;15:R16.

4 Goldberg R, Dennen P: Long-term outcomes of acute kidney injury. Adv Chronic Kidney Dis 2008;15:297-307.

5 McIlroy DR, Argenziano M, Farkas D, et al: Incorporating oliguria into the diagnostic criteria for acute kidney injury after on-pump cardiac surgery: impact on incidence and outcomes. J Cardiothorac Vasc Anesth 2013; 27:1145-1152.

6 Hansen MK, Gammelager H, Mikkelsen MM, et al: Post-operative acute kidney injury and five-year risk of death, myocardial infarction, and stroke among elective cardiac surgical patients: a cohort study. Crit Care 2013;17:R292.

7 Pickering JW, James MT, Palmer SC: Acute kidney injury and prognosis after cardiopulmonary bypass: a metaanalysis of cohort studies. Am J Kidney Dis 2015;65:283-293.

8 Swaminathan M, Hudson CC, Phillips-Bute BG, et al: Impact of early renal recovery on survival after cardiac surgery-associated acute kidney injury. Ann Thorac Surg 2010;89:1098-1104.

9 Karkouti K, Wijeysundera DN, Yau TM, et al: Acute kidney injury after cardiac surgery: focus on modifiable risk factors. Circulation 2009;119:495-502.

10 Leacche M, Winkelmayer WC, Paul S, et al: Predicting survival in patients requiring renal replacement therapy after cardiac surgery. Ann Thorac Surg 2006;81:1385-1392.

11 Lopez-Delgado JC, Esteve F, Torrado H, et al: Influence of acute kidney injury on short- and long-term outcomes in patients undergoing cardiac surgery: risk factors and prognostic value of a modified RIFLE classification. Crit Care 2013;17:R293.

12 Hobson CE, Yavas S, Segal MS, et al: Acute kidney injury is associated with increased long-term mortality after cardiothoracic surgery. Circulation 2009;119:2444-2453.

13 Li SY, Chen JY, Yang WC, et al: Acute kidney injury network classification predicts in-hospital and long-term mortality in patients undergoing elective coronary artery bypass grafting surgery. Eur J Cardiothorac Surg 2011;39:323-328.

14 Foley RN, Parfrey PS, Sarnak MJ: Clinical epidemiology of cardiovascular disease in chronic renal disease. Am J Kidney Dis 1998;32(suppl 3):S112-S119.

15 McClellan WM, Anson C, Birkeli K, et al: Functional status and quality of life: predictors of early mortality among patients entering treatment for end stage renal disease. J Clin Epidemiol 1991;44:83-89.

16 Roques F, Michel P, Goldstone AR, et al: The logistic EuroSCORE. Eur Heart J 2003;24:881-882.

17 Levey AS, Stevens LA, Schmid CH, et al: A new equation to estimate glomerular filtration rate. Ann Intern Med 2009;150:604-612.

18 Teo BW, Xu H, Koh YY, et al: Estimating kidney function in a multiethnic Asian population with multiple filtration markers. Am J Kidney Dis 2012;60:500-502.

19 Bagshaw SM, Uchino S, Bellomo R, et al: Timing of renal replacement therapy and clinical outcomes in critically ill patients with severe acute kidney injury. J Crit Care 2009;24:129-140. 
Lim et al.: Long-Term Risk of Progressive Chronic Kidney Disease in Patients with

Severe Acute Kidney Injury Requiring Dialysis after Coronary Artery Bypass Surgery

20 Mehta RL, Kellum JA, Shah SV, et al: Acute Kidney Injury Network: report of an initiative to improve outcomes in acute kidney injury. Crit Care 2007;11:R31.

21 Rosner MH, Okusa MD: Acute kidney injury associated with cardiac surgery. Clin J Am Soc Nephrol 2006;1: 19-32.

22 Srivastava V, D'Silva C, Tang A, et al: The impact of major perioperative renal insult on long-term renal function and survival after cardiac surgery. Interact Cardiovasc Thorac Surg 2012;15:14-17.

23 Luckraz H, Gravenor MB, George R, et al: Long and short-term outcomes in patients requiring continuous renal replacement therapy post cardiopulmonary bypass. Eur J Cardiothorac Surg 2005;27:906-909.

24 Ishani A, Nelson D, Clothier B, et al: The magnitude of acute serum creatinine increase after cardiac surgery and the risk of chronic kidney disease, progression of kidney disease, and death. Arch Intern Med 2011;171: 226-233.

25 Ryden L, Sartipy U, Evans M, et al: Acute kidney injury after coronary artery bypass grafting and long-term risk of end-stage renal disease. Circulation 2014;130:2005-2011.

26 Garcia-Fernandez N, Perez-Valdivieso JR, Bes-Rastrollo M, et al: Timing of renal replacement therapy after cardiac surgery: a retrospective multicenter Spanish cohort study. Blood Purif 2011;32:104-111.

27 Shum HP, Chan KC, Kwan MC, et al: Timing for initiation of continuous renal replacement therapy in patients with septic shock and acute kidney injury. Ther Apher Dial 2013;17:305-310.

28 Chou YH, Huang TM, Wu VC, et al: Impact of timing of renal replacement therapy initiation on outcome of septic acute kidney injury. Crit Care 2011;15:R134.

29 Doi K, Yuen PS, Eisner C, et al: Reduced production of creatinine limits its use as marker of kidney injury in sepsis. J Am Soc Nephrol 2009;20:1217-1221.

30 Go AS, Parikh CR, Ikizler TA, et al: The assessment, serial evaluation, and subsequent sequelae of acute kidney injury (ASSESS-AKI) study: design and methods. BMC Nephrol 2010;11:22. 